\title{
Photographische Beobachtungen des Planeten 1901 GQ.
}

\begin{tabular}{|c|c|c|c|c|c|c|c|}
\hline $\begin{array}{c}\text { I9OI } \\
\text { Aug. I } 7\end{array}$ & $\begin{array}{l}\text { M. Z. Heid. } \\
10^{\mathrm{h}} 27^{\mathrm{m}} \cdot 7\end{array}$ & $\begin{array}{c}\Delta \alpha \\
+1^{m} 57^{\mathrm{s}} \cdot 79\end{array}$ & $\begin{array}{c}\Delta \delta \\
+9^{\prime} \text { I } 4{ }^{\prime \prime} 3\end{array}$ & $22^{\mathrm{h}} 4^{\alpha \text { app. }}{ }^{\mathrm{m}} 7^{\mathrm{s}} \mathrm{I} 7$ & $\begin{array}{c}\text { ס app. } \\
+6^{\circ} 22^{\prime} 48.7\end{array}$ & $\begin{array}{l}\text { Red. ad 1. app. } \\
+4.02+25 \text {.० }\end{array}$ & $\begin{array}{l}\text { Beob. } \\
\text { Carnera }\end{array}$ \\
\hline 18 & 1210.2 & +13.67 & +916.4 & $2240 \quad 13.06$ & +62251.0 & $+4.03+25.2$ & 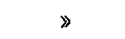 \\
\hline
\end{tabular}

Die Platten sind von Herrn Aug. Kopff nach der Distanzmethode ausgemessen worden.

Heidelberg, Igo I Oct. I I.

Für den abwesenden Vorstand: Dr. Luigi Carnera.

\section{Photographische Beobachtungen von kleinen Planeten}

auf dem Astrophysikalischen Observatorium Königstuhl.

\begin{tabular}{l|}
\hline Planet \\
\hline $\begin{array}{c}\text { (94) Aurora } \\
\text { (167) Urda }\end{array}$ \\
(334) Chicago \\
\\
(390) Alma \\
(1 I) Parthenope \\
(274) Philagoria \\
(49) Pales \\
(108) Hecuba \\
(32 1) Florentina
\end{tabular}

Heidelberg, 1901 Nov. I3.

\begin{tabular}{|c|c|c|c|c|c|c|c|c|c|c|}
\hline 1901 & M.Z.Hei. & $\alpha$ & $\delta$ & Bb. & Pla & I 901 & M.Z.Hei. & $\alpha$ & $\delta$ & $\mathrm{Bb}$. \\
\hline 2 & $7^{\mathrm{h}} 33^{\mathrm{m}} \cdot 4$ & $1^{h} 4^{h} m_{2}$ & $+16^{\circ} 5^{6}$ & $\mathrm{C}$ & 2) $E$ & Nov. 6 & $12^{\mathrm{h}} 5^{6^{\mathrm{m}}} \cdot 5$ & $4^{\mathrm{h}} 58^{\mathrm{m}} \cdot 0$ & $+13^{\circ} 3^{8^{\prime}}$ & $w$ \\
\hline Nov, 2 & 10 7.4 & 344.1 & +1645 & $\mathrm{C}$ & & I 2 & I I 50.1 & 454.6 & +1325 & C \\
\hline & 933.8 & 342 & $+164^{\circ}$ & C & $\left(8_{7}\right)$ Sylv & Nov. 6 & 1459.8 & 445.4 & +1937 & C \\
\hline Nov. 2 & Io 7.4 & 342 & +1325 & $\mathrm{C}$ & $(419) A$ & Nov. 6 & I 459.8 & 3.4 & +2050 & C \\
\hline 3 & I I 32.5 & 342 & +1323 & $\mathrm{C}$ & $(455) \mathrm{Br}$ & Nov, 6 & I 459.8 & 8.2 & +1814 & C \\
\hline & $\begin{array}{ll}9 & 33.8\end{array}$ & 34 & +1322 & C & & I 2 & I I 50.1 & 3.2 & +1837 & C \\
\hline Nov. 3 & $\begin{array}{ll}8 & 16.4\end{array}$ & 340.4 & +3822 & $\mathrm{~K}$ & I $\mathrm{H}$ & fov. 6 & 1459.8 & $7 \cdot 3$ & +20 II & $\mathcal{U}$ \\
\hline Nov. 3 & I I 13.4 & $\begin{array}{ll}2 & 24\end{array}$ & +6 & $\mathrm{~K}$ & $1 \mathrm{H}$ & V. 12 & & 0.9 & +230 & w \\
\hline Nov. 3 & I I 13.4 & $2 \quad 16.3$ & +9 & $\mathrm{~K}$ & $1 \mathrm{H}$ & Nov. 12 & 84 I.I & 4.2 & +125 & $\mathrm{C}$ \\
\hline 4 & I2 30.6 & 358.6 & +25 & C & $01 \mathrm{~F}$ & v. 12 & 841.1 & 4.9 & +127 & 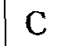 \\
\hline 4 & I 230.6 & 340.0 & +25 & C & OI $\mathrm{H}$ & . 12 & $\begin{array}{ll}9 & 48.4\end{array}$ & $\begin{array}{lll}2 & 21.7\end{array}$ & +750 & W \\
\hline Nov. 4 & 1230.6 & 333.7 & +1957 & C & $\begin{array}{l}1901 \mathrm{H} \\
(404) \mathrm{A}\end{array}$ & $\begin{array}{l}\text { Nov, I } 2 \\
\text { Nov. } 12\end{array}$ & $\begin{array}{rr}9 & 48.4 \\
11 & 50.1\end{array}$ & $\begin{array}{ll}2 & 31.7 \\
4 & 55.2\end{array}$ & $\begin{array}{l}+541 \\
+1317\end{array}$ & W \\
\hline
\end{tabular}

$$
\mathrm{C}=\text { Carnera, } \mathrm{K}=\text { Kopff, } \mathrm{W}=\text { Wolf } \text {. }
$$

M. Wolf.

1) Grösse 11.5. - 2) = 1901 HG. - 3) Von Prof. Wolf als wahrscheinlich identisch mit (367) Amicitia angegeben. Da ich aber den Planeten eher für neu halte, mag er zunächst mit $1901 \mathrm{HK}$ bezeichnet werden. $K r .-{ }^{4}$ ) Gr. I1.5. - 5 ) Neuer Planet Carnera Gr. II.3,

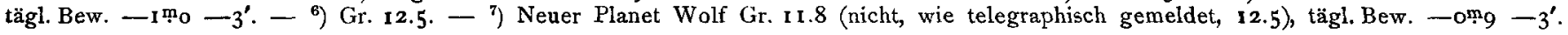

\section{Nova (3.1901) Persei.}

In Betreff des die Nova Persei umgebenden Nebels (vgl. Wolf, A. N. $373^{6}$ p. 256 , und Ritchey, Nebulosity about Nova Persei, Astrophys. Journal, Oct. 1901) sind als Ergänzung zu der in A. N. 3748 mitgetheilten Nachricht noch die folgenden Telegramme von Prof. Pickering eingegangen:

Nov. 1 2: "Ritchey states photograph Yerkes Observatory Nov. $9^{\text {th }}$ confirms large motion of nebula near Nova.\&

Nov. I 2: * Ritchey finds nova nebula probably expanding in all directions. \&

$K r$.

(380) Fiducią. Corr. der Ephemeride (Veröff. R. I. 1 5): Nov. $5-\mathbf{1}^{\mathrm{m}} 55^{\mathrm{s}}-\mathbf{1}$ 1:2 Gr. 1 2.0. Abetti.

(391) Ingeborg. Der Planet ist nachträglich von Prof. Wolf auf einer Platte vom 8. Juli d. J., $\mathbf{I}^{\mathrm{h}} \mathbf{1} \mathbf{I}^{\mathrm{m}} \cdot 3 \mathrm{M} . \mathrm{Z}$ Heidelberg wie folgt aufgefunden worden: $\alpha=23^{\mathrm{h}} 28^{\mathrm{m}} \cdot 9 \delta=+30^{\circ} \mathrm{II}^{\prime}(1855.0)$. Die Correction der Ephemeride in A. N. $374^{6}$ beträgt nach Prof. Abetti: Nov. 6 - r 2.0 - r:o Gr. I 1.5.

Planet 1901 HJ. Nov. I $512^{\mathrm{h}} 57^{\mathrm{m}}$ 。 Düsseldorf RA. $=4^{\circ} 4^{\prime} 30^{\prime \prime} \mathrm{PD} .=88^{\circ} 4^{\prime} \mathrm{I}^{\prime} 6^{\prime \prime}$ Gr. 11.2. W. Luther.

Personalnotizen. Prof. Pietro Tacchini hat das Amt eines Directors des R. Ufficio centrale di Meteorologia e Geodinamica al Collegio Romano niedergelegt; zu seinem Nachfolger ist Prof. Luigi Palazzo ernannt worden. Die ebenfalls im Collegio Romano befindliche kgl. Sternwarte wird auch weiterhin unter der bewährten Leitung von Prof. Tacchini verbleiben. Zum Vorstand der Sternwarte des k. und k. hydrographischen Amtes in Pola ist der k, und k. Corvettenkapitän $K$. Koss ernannt worden.

\footnotetext{
Inhalt zu Nr. 3750. T.F.F. See. Preliminary Investigation of the Diameter of Mars. 97. - A. Berberich. Planet (455) Bruchsalia. I 3 . - L. Car. nera. Photographische Beobachtungen des Planeten I90I GQ. I I 5. - M. Wolf. Photographische Beobachtungen von kleinen Planeten. 1 I5. - Nova (3.I90I) Persei. I I5. - Mittheilungen über kleine Planeten. I I5. - Personalnotizen. I I5. 